\title{
Acceso a bases de datos de museos y bibliotecas: el proyecto BABEL
}

\author{
José María Martínez, \\ Guillermo Cisneros, \\ Jesús Bescós
}

Grupo de Tratamiento de Imágenes Departamento de Señales, Sistemas y Radiocomunicaciones E.T.S. Ingenieros de Telecomunicación

Universidad Politécnica de Madrid

\section{INTRODUCCIÓN}

Las tecnologías digitales dan lugar a cambios en el modo de desempeñar los papeles tradicionales de los museos, archivos y bibliotecas: conservación de los fondos, investigación sobre esos fondos y diseminación tanto de los fondos como de los resultados de las investigaciones llevadas a cabo sobre esos fondos.

Respecto a la conservación, las tecnologías digitales se aplican mayoritariamente a la creación, actualización y administración de bases de datos. Aunque no es el objeto de este trabajo, también estas tecnologías se aplican a otros ámbitos de conservación e incluso de investigación; por ejemplo, el tratamiento digital de imágenes puede ser de gran ayuda a la hora de planificar restauraciones, descubrir capas ocultas, etc. El acceso a bases de datos para recuperación y modificación de la información relativa a los fondos es también una aportación fundamental para la investigación y no sólo para la conservación, ya que permite un acceso más ágil y a una mayor cantidad de información diseminada por todo el mundo (gracias al acceso a través de redes de comunicación).

Por último, el papel de difusores de los fondos y de la información asociada a ellos se mejora (tanto por las nuevas facilidades de accesibilidad, como por las nuevas formas de expresión) mediante el acceso (recuperación) de información de las bases de datos.

Actualmente, la mayor parte de los museos, archivos y bibliotecas se han limitado a ser simplemente proveedores de información, esto es, solamente se encargaban de mantener para uso interno (en el mejor de los casos) archivos digitales multimedia. El papel de proporcionar las aplicaciones sobre esos contenidos era llevado a cabo por otros actores que tenían los conocimientos necesarios para llevar a cabo las aplicaciones que desplegasen esa información almacenada en los archivos. Las aplicaciones pueden ir desde simples (o complejos) catálogos multimedia hasta exposiciones virtuales (libres o guiadas), programas educacionales, ... Un aspecto importante a tener en cuenta son los distintos usuarios a los que puede ir dirigida una aplicación concreta, o los diversos niveles que puede haber dentro de una aplicación para los diversos usuarios (e.g., conservadores, bibliotecarios, público en general, niños, ...).

La provisión de aplicaciones y servicios sobre los contenidos se puede llevar a cabo a través de diversos medios. El más simple e inmediato son los libros digitales, categoría en la que se encuentran los CD-ROMs y los webs con páginas HTML estáticas. Se trata de escribir libros con las nuevas tecnologías digitales como soporte. Este medio de desarrollo de aplicaciones tiene su campo bien delimitado, como por ejemplo la creación de catálogos de exposiciones. Sin embargo, las tecnologías digitales permiten otra forma más potente y dinámica para la provisión de aplicaciones y servicios, lo que podríamos denominar aplicaciones sobre "almacenes digitales, término que generaliza el ya extendido de digital libraries. Estos almacenes digitales son aplicaciones desarrolladas sobre bases de datos, que proporcionan múltiples ventajas sobre los libros digitales: son aplicaciones más baratas de mantener y actualizar (si bien su desarrollo e implantación inicial es más costosa), permiten acceder siempre a la información actualizada (siempre y cuando ésta se actualice), permite desarrollar aplicaciones para el soporte a la creación de libros digitales (por parte de conservadores e investigadores), y si se desarrollan adecuadamente permiten la reutilización de nuevas aplicaciones sobre bases de datos existentes y de nuevas bases de datos (contenidos) para aplicaciones existentes.

La aparición y posterior popularidad de Internet proporciona conectividad a nivel mundial (global), lo que nos permite hablar de teleservicios. Esta posibilidad de acceso desde cualquier lugar da un claro valor añadido tanto a las aplicaciones de libros digitales, como a las de almacenes digitales, ya que se puede acceder a la aplicación sin tener que pasar por los trámites intermedios entre el editor y el usuario final (cadena de distribución). Adicionalmente al acceso desde cualquier lugar, las aplicaciones de almacenes digitales tienen la posibilidad de proporcionar otros valores añadidos, como el acceso común (incluso homogéneo) a bases de datos heterogéneas.

No se debe confundir Internet con World Wide Web. Si bien es ésta última la que a llevado a Internet a sus altísimas cotas de popularidad, no es el único servicio (véase el caso del correo electrónico) que se puede utilizar sobre la infraestructura de red y conectividad global a través de protocolos estándar. Por otra parte, WWW es "sencillo" de usar, 
pero carece (ipor ahora?) de la versatilidad de aplicaciones ad-hoc, que utilizarán Internet para la interconectividad. Esto hace que hoy en día aún tenga sentido seguir desarrollando aplicaciones para servicios concretos en Internet que no están basadas en WWW.

\section{PROYECTOS ANTERIORES}

En esta sección se describen brevemente proyectos anteriores relacionados con el acceso a bases de datos de museos.

\section{European Musems Network}

El proyecto EMN (European Museums Network) fue financiado por la Unión Europea dentro de su programa RACE entre los años 1989 y 1992.

El proyecto desarrolló un almacén digital que era actualizado a través de Internet, mientras que la consulta de los contenidos por parte del público se llevaba a cabo sobre una serie de terminales conectados a una red de área local en cada museo participante. El servidor de cada museo se encontraba conectado a esta red de área local y recibía las actualizaciones del servidor central a través de Internet.

Se desarrollaron tres aplicaciones:

- Multimedia Data Acquisition (MDA), que permitía la creación de Fondos Digitales por parte de conservadores de los museos

- Aplicación Visitor, que permitía la consulta de la base de datos del EMN y la navegación entre los distintos objetos a través de palabras clave

- Aplicación Upgrade (Central MDA), que permitía al administrador del sistema gestionar los nuevos fondos y modificaciones (e.g., traducciones), comprobar su consistencia, y actualizar la base de datos central, para posteriormente actualizar las réplicas en cada museo.

Tras la finalización del proyecto algunos museos mantuvieron los kioscos interactivos a disposición del público hasta que por problemas de mantenimiento de los equipos originales fueron desmantelados.

Actualmente se está desarrollando el proyecto FENIX, cuya misión es recuperar el sistema cambiando el software de interfaz de usuario para pasarlo de su antiguo interfaz para terminales Macintosh a una interfaz de usuario para WWW. Por ahora se ha realizado la aplicación Visitante de FENIX, que permite el acceso a través del WMW a los fondos multimedia de la base de datos del EMN (http://www.gti.ssr.upm.es/fenix), y la aplicación de adquisición y actualización está comenzado a ser desarrollada.

\section{Remote Acces to Museums Archives}

El proyecto RAMA (Remote Access to Museums Archives) fue también financiado por la Unión Europea en la segunda convocatoria RACE desde 1992 a 1995. Uno de los problemas detectados durante el proyecto EMN fue la reticencia de los conservadores de los museos a crear fondos dentro de unos formatos comunes, ya que les coartaba su expresividad así como su "libertad de cátedra". Por lo tanto, dentro del proyecto RAMA se planteó como punto de partida la no creación de bases de datos comunes a todos los museos, y por lo tanto el respeto a las heterogeneidades de cada institución, e incluso de cada departamento. Así pues, el objetivo del proyecto era la creación de un sistema que permitiese a los museos compartir sus fondos pero sin sacrificar sus estructuras y normas de gestión de sus bases de datos. Se desarrolló un sistema de información que permitía el acceso a bases de datos heterogéneas a través de una aplicación común, manteniendo la infraestructura de cada museo (HW, DBMS, etc..) y preservando las preferencias y particularidades (estructura de base de datos) de cada institución y departamento. Para probar el sistema se desarrolló una aplicación de Tele-investigación. Esta aplicación de Tele-investigación estaba destinada a usuarios profesionales (conservadores e investigadores) y proporcionaba un método unificado para el acceso a las diferentes bases de datos. Como características principales permitía la consulta a través de un mecanismo sencillo, potente y común, la globalización de resultados provenientes de diferentes bases de datos, y el soporte de sesiones, permitiendo almacenar la historia de la búsqueda y preservar la información para reanudar la sesión en momentos posteriores.

Una vez concluido el proyecto, se abandonó el desarrollo posterior del sistema dentro del consorcio. Sin embargo, partiendo de los módulos software desarrollados por nuestro grupo, llevamos a cabo el desarrollo de un sistema completamente español evolucionado a partir de las ideas y experiencias del proyecto RAMA. Este desarrollo dio lugar al sistema MIRA (Multimedia Information Remote Access) y a su aplicación de Teleinvestigación. El cliente de esta aplicación es de libre distribución en Internet', y se puede descargar libremente desde la URL http://www.gti.ssr.upm.es/mira/.

\section{EL PROYECTO BABEL}

BABEL (Servicios Multimedia para museos y bibliotecas vía Internet TEL96-128I financiado por la Comisión Interministerial de Ciencia y Tecnología) es un proyecto encaminado a la creación de una red de museos y bibliotecas en España que haga posible la teleconsulta de sus bases de datos a través de Internet. El proyecto surge como demostración de los resultados obtenidos en proyectos anteriores y para servir de escaparate para posibles nuevas iniciativas en Europa. El alias BABEL indica la heterogeneidad existente, tanto en la diversidad de plataformas (SW, HW, DBMS), como en la diversidad en las estructuras de datos específicas (fichas de inventario, catálogo). Se trata de proporcionar la tele-consulta de bases de datos heterogéneas a través de un sistema común, que:

- permita la reutilización de las bases de datos existentes, respetando sus heterogeneidades,

- proporcione un Manual de Usuario único para el acceso a las diferentes bases de datos, 
- garantice el acceso mediante una pareja única por usuario de nombre de usuario y clave (login y password) para acceder a las diversas bases de datos; no obstante, el mismo login tiene diferentes perfiles de acceso a la información en las diferentes bases de datos, y por lo tanto diferentes permisos de acceso a campos determinados de las fichas en cada museo (que pueden ser ocultados a determinados perfiles). El sistema contempla dos aplicaciones de acceso: la aplicación Tele-investigación de MIRA para usuarios profesionales y el acceso WWW (a través de la pasarela CATALOG) para el gran público.

\section{Participantes}

Las instituciones que forman parte del consorcio del proyecto BABEL son las siguientes, agrupadas según su papel:

- Proveedores de contenidos y usuarios: Museo Arqueológico Nacional, Fundación Centro Nacional del Vidrio Real Fábrica de Cristales de La Granja, , Biblioteca de la Universidad Carlos III de Madrid, y Servei de Museus de la Generalitat de Catalunya. El Museo Nacional del Prado y Patrimonio Nacional - Palacio Real- abandonaron el proyecto a finales de 1998 por cuestiones internas.

- Proveedores de Tecnología: Universidad Politécnica de Madrid (Grupo de Tratamiento de Imágenes)

- Entes Promotores Observadores: Telefónica (Telefónica Sistemas) y Ministerio de Educación y Cultura (Subdirección General de Tratamiento de la Información y Subdirección General de Museos Estatales - Comisión de Normalización), Caja de Madrid, Hewlett-Packard, e Informix.

Adicionalmente existe un Grupo de Interés, que agrupa a aquellas instituciones interesadas en formar parte del proyecto BABEL bien como usuarios o como futuros proveedores de contenidos.

\section{Objetivos}

Cuatro son los objetivos principales que se plantea el proyecto BABEL. El primero de ellos es la creación de una red de museos y bibliotecas en España, que permita la teleconsulta de sus bases de datos a través de Internet a usuarios profesionales y al gran público. Para cumplir este objetivo, y partiendo de los productos software MIRA y CATALOG se desarrollan las pasarelas requeridas para las bases de datos de los participantes $y$, adicionalmente, de algunos miembros del Grupo de Interés. El proyecto BABEL no está pensado como un proyecto de desarrollo, sino como un piloto de aplicación de tecnología, previamente desarrollada, dentro de un marco real por lo que se limita el desarrollo software al máximo. No se trata de crear una red solamente a nivel telemático, sino que se potencia la creación de una red humana para una mayor colaboración entre las diversas instituciones culturales participantes y el Grupo de Interés.

El segundo objetivo del proyecto BABEL es el preparar el camino para la resolución de las carencias en el entorno de museos y bibliotecas en España, promocionando la existencia de almacenes digitales multimedia (bases de datos), la populación de estos almacenes digitales, y el acceso a Internet desde las instituciones (popularizando su uso), así como el acceso desde Internet a las instituciones para darlas a conocer (difusión de fondos).

El tercer objetivo es la creación de un Museo y una Biblioteca Virtual (Punto de Información Común), esto es, una base de datos que permita un acceso común a los descriptores de las diferentes bases de datos de la red BABEL y un "encaminamiento" transparente hacia las fichas completas.

Como último objetivo, el proyecto BABEL se propone ser uno de los pioneros en el campo de los nuevos papeles que museos, bibliotecas y archivos están llamados a desarrollar de cara al siglo XXl. Este papel de pionero queda avalado por el interés despertado, habiendo sido presentado el proyecto en diversos foros tanto nacionales como internacionales.

\section{El despliegue de BABEL}

Para llevar a cabo el despliegue de BABEL, el programa del proyecto consiste en llevar a cabo un plan en diversos pasos. El primer paso consiste en verificar y subsanar la conexión a Internet de todas las instituciones participantes, para pasar a la creación de los sitios WWW de las instituciones participantes (museos y bibliotecas) que no disponían de ellos.

El segundo paso consiste en la organización las bases de datos existentes, así como la creación de aquellas que no estaban en fase de creación antes de iniciar el proyecto. Una vez que existe la base de datos de cada institución en explotación interna (esto es, para uso por parte de aplicaciones propietarias de cada institución), se pasa a instalar el software proporcionado dentro del proyecto BABEL: el servidor MIRA y la aplicación de Tele-investigación, y la pasarela CATALOG para acceso a las bases de datos a través de WWW. La aplicación Tele-investigación de MIRA está destinada al usuario profesional (conservadores, bibliotecarios, investigadores, ...), y aparte de la instalación del software común se debe llevar a cabo el desarrollo de la pasarela a la base de datos de la institución. El acceso a las bases de datos a través WWW para el gran público requiere la instalación y personalización de la pasarela CATALOG, para permitir consultar la base de datos de un modo más sencillo, bien mediante consultas (para grandes bases de datos) o navegación (para bases de datos más sencillas).

El paso siguiente consiste en el desarrollo del Museo y Biblioteca Virtual. Este desarrollo implica la creación de la base de datos con una serie de campos comunes a rellenar con datos procedentes de las diversas bases de datos, así como la instalación del software de MIRA y CATALOG, como si de una institución más se tratase.

El diseño y desarrollo de MIRA y CATALOG permite la independencia entre la provisión de información y de servicio (aplicación) de modo que nuevas bases de datos pueden ser "enchufadas" fácilmente a aplicaciones y servicios existentes, así como nuevas aplicaciones y servicios pueden ser desarrolladas sobre bases de datos existentes.

Los componentes principales de los Sistemas de Información MIRA y CATALOG son: 
- servidores de base de datos, que actúan como pasarelas a las diversas bases de datos, presentando una interfaz común al resto del sistema, esto es, la abstracción de una base de datos única

- un núcleo funcional, que actúa como plataforma para el soporte al desarrollo de aplicaciones, incluyendo las comunicaciones sobre Internet.

- servidores de aplicación (MIRA y CATALOG), que se encargan de proporcionar las funcionalidades específicas de cada aplicación (por el momento, Tele-investigación de MIRA y consulta de CATALOG)

- interfaz de usuario de la aplicación, que proporciona los módulos encargados de la presentación e interacción con el usuario, bien mediante interfaces Windows ad-hoc (Tele-investigación) o HTML para navegadores WWW (consulta a través de CATALOG).

\section{Estado actual}

Actualmente todos los participantes a excepción del Servei de Museus disponen de sitios WWW. El acceso a las bases de datos es completo en el caso del Museo Arqueológico Nacional (más de 40000 fichas accesibles), mientras que en el caso del Museo del Vidrio y la Biblioteca de la Universidad Carlos III, se está pendiente de instalar en las instituciones, pudiéndose acceder a una copia reducida de sus bases de datos. El Servei de Museus está pendiente de finalizar el desarrollo de sus bases de datos.

Se puede acceder a los diversos sitios y servicios desde el sitio WWW del proyecto BABEL en la siguiente dirección htp://www.gti.ssr.upm.es/babel/. Para obtener libre- mente el cliente de la aplicación MIRA de Tele-investigación se puede acceder directamente a la dirección http://www.gti.ssr.upm.es/mira/.

\section{CONCLUSIONES}

En este artículo hemos presentado en primer lugar las ventajas que proporciona el desarrollo de aplicaciones que operan sobre bases de datos para el acceso a información proporcionada por instituciones culturales, para posteriormente presentar dos proyectos europeos que dieron lugar al lanzamiento del proyecto BABEL. El proyecto BABEL tiene como objetivo principal la creación de una red de museos y bibliotecas en España que permite el acceso a través de Internet a aplicaciones desarrolladas sobre la información almacenada en sus bases de datos. Tanto para las aplicaciones de acceso de profesionales como para las de acceso de gran público se proponen sistemas comunes para acceder a bases de datos heterogéneas, respetando las bases de datos existentes y su estructura interna de datos. Se propone también la creación de un Museo y Biblioteca Virtual (Punto de Información Común), que permita el acceso simultáneo a descriptores de fichas procedentes de las diversas bases de datos, así como el "encaminamiento" automático y transparente al usuario a las fichas completas residentes en los archivos digitales del correspondiente Museo o Biblioteca.

Como resultado final se pretende crear una infraestructura (tecnológica y humana) para una red de provisión de servicios que permita, gracias a un diseño y desarrollo adecuados, que nuevas bases de datos puedan ser "enchufadas" fácilmente a aplicaciones existentes, y que nuevas aplicaciones puedan ser desarrolladas sobre bases de datos existentes.

\section{Bibliografía}

GUILLERMO CISNEROS, JESÚS BESCÓS, JOSÉ M. MARTÍNEZ, "Telemuseum services via Internet: present and future", Information Services and Use, 16 (2), Agosto 1996.

JOSÉ M. MARTÍNEZ, FRANCISCO MORÁN, "CATALOG: a WWW gateway for RDBMS", en WebNet96, AACE 1996

JOSÉ M. MARTíNEZ, JESÚS BESCÓS, GUILLERMO CISNEROS, "Servicios Multimedia en Museos y Bibliotecas vía Internet", Libro de Ponencias de Mundo Internet 97, Madrid, Febrero 1997

JOSÉ M. MARTÍNEZ, FRANCISCO MORÁN, "Generación de hiperbases en Internet", Libro de Ponencias de Mundo Internet 97, Madrid, Febrero 1997
GUILLERMO CISNEROS, JOSÉ M. MARTÍNEZ, JESÚS BESCÓs, "The BABEL Project: a Museums and Libraries Network", Proceedings of Museum and the Web 1998, Toronto, April 1998.

JOSÉ M. MARTÍNEZ, JESÚS BESCÓS, GUILLERMO CISNEROS, "Introducing MIRA", en Research and Advanced Technology for Digital Libraries, Lecture Notes in Computer Science, vol. 1513, Springer-Verlag, 1998

JOSÉ M. MARTíNEZ and SIXTO HERNÁNDEZ, "Introducing PHOENIX", en WebNet98, AACE 1998 